11 | 2018

Écrire le fleuve Congo après Conrad

\title{
Hors Conrad : Congo Eza
}

Joëlle Sambi

\section{OpenEdition \\ Journals}

Édition électronique

URL : https://journals.openedition.org/coma/2998

DOI : $10.4000 /$ coma.2998

ISSN : 2275-1742

Éditeur

Institut des textes \& manuscrits modernes (ITEM)

Référence électronique

Joëlle Sambi, «Hors Conrad : Congo Eza », Continents manuscrits [En ligne], 11 | 2018, mis en ligne le

15 octobre 2018, consulté le 14 janvier 2023. URL : http://journals.openedition.org/coma/2998 ; DOI https://doi.org/10.4000/coma.2998

Ce document a été généré automatiquement le 14 janvier 2023.

\section{(c) (i) (2)}

Creative Commons - Attribution - Pas d'Utilisation Commerciale - Pas de Modification 4.0 International - CC BY-NC-ND 4.0

https://creativecommons.org/licenses/by-nc-nd/4.0/ 


\title{
Hors Conrad : Congo Eza
}

\author{
Joëlle Sambi
}

\section{«Conrad me revient par la rampe»}

1 Lorsqu'on m'a demandé de préparer cette intervention, je me suis dit que Conrad et son ouvrage Au cour des ténèbres étaient assez peu présents dans mon travail et bien loin de la littérature à laquelle je m'intéresse. Il a fallu m'y replonger pour m'apercevoir qu'il était en fait vraiment très proche. Il $\mathrm{y}$ a parfois des lectures qui nous imprègnent et dont on ne saisit pas immédiatement l'importance...

2 Je suis née à Bruxelles et j'y ai commencé mes premières classes maternelles. Enfant, j'ai donc appris ce que les petits Belges apprenaient à cette époque-là. Ensuite, je pars vivre avec ma famille à Kinshasa et ma mère me met à l'école belge. Pendant toutes mes études primaires, jusqu'à l'âge de onze ans environ, je découvre l'histoire et la géographie du monde et de la Belgique en particulier. Pas celle du Zaïre. J'apprends même la Brabançonne avant la Zaïroise. Je grandis comme ça sous la dictature de Mobutu, comme un arbre planté dans sa propre terre mais qui aurait du mal à y plonger ses racines. À un moment donné, en raison de quelques soubresauts politiques dont j'ignore véritablement les tenants et les aboutissants, il est question pour tous les élèves congolais - pour les ressortissants zaïrois, y compris ceux qui fréquentent les écoles congolaises, françaises ou belges - de suivre des cours de civisme afin que tous aient une connaissance suffisante de leur propre histoire et de leur géographie, bref de leur culture. Ainsi, à la fin des classes, en soirée, environ deux fois par semaine, je me retrouve dans ce cours avec mes autres camarades congolais. Je découvre alors à onze ans que je ne sais pas où se situe sur la carte du Zaïre la ville de Kinshasa dans laquelle je vivais depuis des années!

3 À l'époque, si vous me posiez une question sur les provinces en Belgique, ou sur la royauté, je pouvais aisément vous en parler; si vous me posiez la même question sur le Zaïre, pas certain que je sois en mesure de parler d'autre chose que de Kinshasa et du quartier où je grandissais. 
4 Au début des années nonante (1990), peu à peu, troubles et pillages adviennent et se multiplient. Les écoles ferment, c'est une année blanche pour la plupart des écoliers. Ma mère me met à l'internat, dans une école conventionnée et catholique, où j'en apprends un peu plus sur l'histoire, la géographie, la littérature africaine et le latin - je peux réciter Quosque tandem abutere patientia nostra Catilina ?', « jusqu'à quand abuserastu ainsi de notre patience, Catilina? »-, mais je ne sais toujours pas parler de l'histoire du Congo. Du moins, il n'y a pas beaucoup d'incitants ni même de raisons particulières à la connaître. La dictature, voilà l'histoire. Nous étions en plein dedans, tous, quels que soient nos âges et nos classes sociales, à la fois acteurs passifs et sujets ébahis d'une politique nationale en pleins bouleversements. Difficile pour moi de comprendre, de saisir tous les enjeux de ce pays devenu (aussi) mien. Et si, adolescente, je plonge tout entière dans la lecture pour échapper à ce que je ne comprends pas, les bibliothèques quasi inexistantes sont vides (de sens) pour moi ou l'adhésion trop onéreuse. Je m'intéresse à la fiction, consciente du manque et d'un accès difficile à l'information. De toute façon, comme je commence à écrire, l'Histoire ne m'intéresse pas. Je préfère la fiction, raconter des histoires, j'écris donc des nouvelles, des poèmes, mais l'histoire de mon pays - celui dans lequel je vis et je grandis - ne m'intéresse pas. À cette époque-là, je lisais les auteurs qui, pour moi, parlaient de vrais gens - c'est-à-dire de celles que je pouvais croiser au quotidien. Zamenga Batukezanga ${ }^{2}$, par exemple, que j'adorais parce qu'il racontait la vie des couples, des quartiers, des histoires ordinaires sur mes contemporains - ça pouvait être mon grand-père ou mon arrière-grand-mère - ce n'était pas quelque chose de lointain ou d'incompréhensible comme la colonisation ou la dictature. J'avais peut-être inconsciemment besoin de m'en échapper et d'aller lire d'autres auteurs.

5 À l'école, aussi j'ai découvert beaucoup d'auteurs africains, et le livre qui m'a le plus marquée est $\hat{O}$ pays, mon beau peuple de Ousmane Sembène. Ce sont plutôt ces auteurs-là qui m'ont marquée, mais pas de traces de Conrad ni de Au cour des ténèbres, jusqu'à ce que je rentre à Bruxelles pour faire des études de journalisme à l'Université Libre de Bruxelles (ULB). Là, le déracinement... mais en est-ce un ? Finalement, je suis née là, en Belgique, j'y reviens... c'est quand même la maison sans être tout à fait la maison... tout cela est un peu compliqué.... Mais j'ai tout de même quitté le Congo, alors j'ai besoin de savoir d'où je viens. Je me remets donc à m'intéresser à mon histoire. Je vais à la bibliothèque, et je lis Au coeur des ténèbres - plutôt, je le feuillette. Cela m'ennuie profondément, je trouve le livre mal écrit... Cette forêt, cette jungle, ce n'est pas mon Congo : je suis kinoise, la jungle et la forêt, c'est (un peu) vert comme Kinshasa mais c'est tout ! - je ne laisse pas beaucoup de chance au roman. Je tombe ensuite sur Les Fantômes du roi Léopold ${ } I^{3}$ et c'est la révélation. C'est le livre qui m'a permis de me réconcilier avec mon histoire (moi-même?) et d'en apprendre beaucoup plus en 435 pages sur mon passé que pendant toutes mes années d'études au Congo et en Belgique.

Dans cet ouvrage, Adam Hochschild parle des résistants : des Congolais, ni amorphes ni an-historiques, se tiennent debout et tentent de résister du mieux qu'ils peuvent. Je prends conscience que je ne fais pas partie d'une nation de vaincus. Alors, je me redresse et je comprends qu'il y a lieu d'en apprendre davantage sur mon peuple. Je dévore tout ce qui me tombe dans la main et qui parle du Zaïre devenu République Démocratique du Congo. Des articles, des livres, des films, comme Apocalyse now, 
notamment que je regarde en boucle sans jamais faire de lien avec Joseph Conrad, même si je me plonge dans les ténèbres tout doucement, sans m'en rendre compte.

7 C'est par un détour très lointain que je vais (re)venir à Conrad. Un jour, une amie artiste vidéo, Liv Bugge ${ }^{4}$ - «Tu me donnes envie de mourir à la campagne »- me dit: "J'ai besoin que tu lises un texte que j'ai découvert, il est incroyable ", sans préciser lequel. En voici un extrait :

Ces boules rondes n'étaient pas ornementales mais symboliques; elles étaient expressives et déconcertantes, frappantes et troublantes - de quoi nourrir la pensée et aussi les vautours s'il y en avait eu à regarder du haut du ciel. Mais, en tout cas, elles étaient fourmis qui étaient assez entreprenantes pour monter au piquet. Elles auraient été encore plus impressionnantes, ces têtes ainsi fichées, si les visages n'avaient pas été tournés vers la maison. Une seule, la première que j'avais distinguée, regardait de mon côté. Je ne fus pas aussi choqué que vous pouvez le penser. Mon sursaut en arrière n'avait été, réellement, qu'un mouvement de surprise. Je m'étais attendu à voir une boule de bois, comprenez-vous. Je retournais délibérément à la première repérée et elle était bien là, noire, desséchée, ratatinée, les paupières closes. Une tête qui, semblant dormir en haut de ce piquet et avec les lèvres séchées et rentrées qui montraient les dents en une étroite ligne blanche, souriait aussi. Souriait continûment de quelque rêve interminable et jovial dans son sommeil éternel.

Voilà que Conrad me revient par la rampe et je ressens qu'il est alors temps de sérieusement m'y plonger. Je le lis, moins agacée, et contrairement à ma première impression, je trouve que sa manière d'écrire est assez impressionnante tant elle me prend... Sa façon de décrire cette faune, cette flore, ce fleuve... Je peux ressentir la chaleur humide, avoir la paume des mains moite, être presqu'aussi avide que lui de remonter le fleuve. Je dois avouer que je ne suis pas vraiment choquée par ses propos sur les hommes noirs, qui, dans son récit, bien que très présents, sont aussi inexistants. Un paradoxe qui peut-être me laisse de marbre parce que j'ai désormais un regard plus critique et plus détaché quand je lis des choses sur le Congo.

Le livre de Conrad Au cœur des ténèbres va me pousser à collectionner des articles de presse, je découpe et archive tout ce que je trouve sur l'État indépendant du Congo et entame ainsi ma petite bibliothèque personnelle d'archives. Au fil de mes recherches, je trouve un autre livre, qui d'ailleurs va m'inspirer pour le titre de mon deuxième roman en écriture - Du sang sur les lianes ${ }^{5}$. C'est ce livre-là, avec sa phrase sentencieuse " tu vas aller au caoutchouc " qui m'inspire peut-être plus qu'Au cœur des ténèbres et qui me donne la mesure de l'influence de Conrad et de ses écrits sur mon vécu.

Ensuite, pour un atelier d'écriture que je devais animer dans le cadre du séminaire de l'École de Recherche Graphique (ERG) à Bruxelles, «After Empire: Melancholia Or Convivial Culture?", j'ai fait des recherches sur l'Empire et le postcolonial et j'ai découvert un texte publié dans Anthropologie et Sociétés, « Ne pas oublier M. Kurtz. L'attrait de la sauvagerie ${ }^{6} »$. Son auteur, Gilles Bibeau y confronte les perceptions de Conrad par Ngugi wa Thiong' ${ }^{7}$ et Chinua Achebe ${ }^{8}$ qui se demandent si Au coeur des ténèbres peut être qualifié de colonial, postcolonial ou anticolonial? Pour certains, Joseph Conrad serait un écrivain impérial (iste) et donc raciste, colonialiste, pour d'autres, c'est un homme de son temps, vivant dans les travers de ceux-ci et qui pourtant tente de montrer (dénoncer ?), en tout cas donne à voir, un fait, une réalité de son époque qui n'est pas des plus glorieuses. Quant à moi, le débat me paraît incongru car pour moi, Conrad n'est pas plus qu'un auteur euro-centrique qui, même s'il parvient à pointer les dérives du projet colonialiste des empires de l'époque - ce qui est 
tout à son honneur -, demeurait un homme du XIX ${ }^{e}$ siècle incapable de se représenter l'Afrique et les Africains comme des êtres capables de se prendre en charge par euxmêmes.

\section{« Décolonie. Apostasie »}

11 Une des choses qu'on reproche à Conrad est d'écrire sur une situation, un pays et des gens avec des épithètes stigmatisantes, racistes, mais, finalement, le geste premier était intéressant et l'intention de dénonciation était louable. Mais était-ce seulement son intention?

12 L'atelier que j'ai dirigé pour l'ERG ${ }^{9}$ en 2017 s'intitulait «Décolonie. Apostasie » et s'étendait sur deux jours. On y a parlé de colonie et postcolonie. Il ne s'agissait pas, pour moi, de trancher la question du Conrad politique ou non. Ce qui m'intéressait et m'intéresse toujours dans l'œuvre de Conrad, ce sont les pièges du langage (et peut-être de la pensée ?) dans lesquels l'auteur tombe et qui amènent à douter de son efficacité...

Photographie de la performance « Décolonie - apostasie »

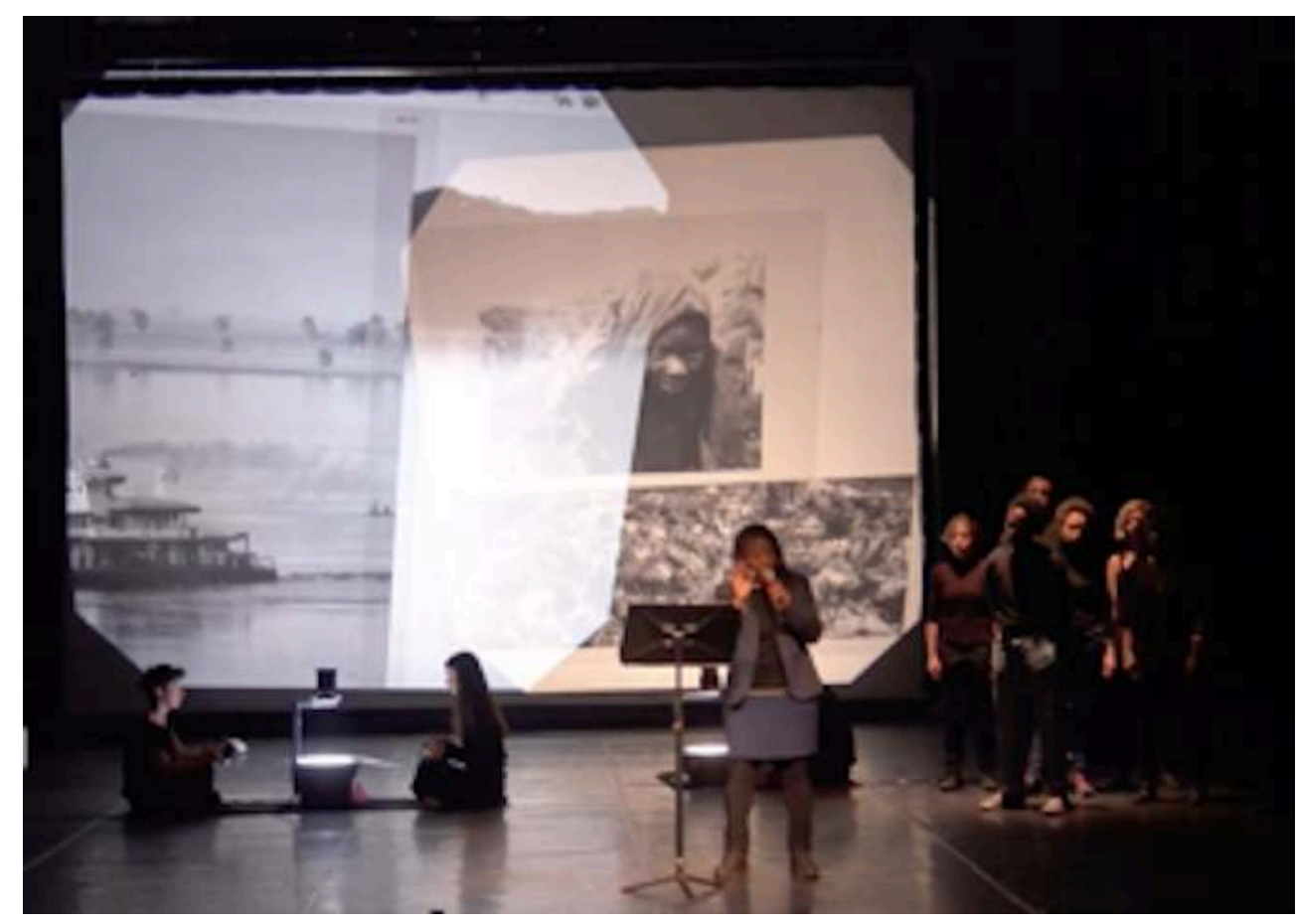

«Décolonie. Apostasie " était à la fois un atelier d'écriture et une tentative de performance-slam en crash test complet. La question consistait à savoir si l'avènement d'un monde postcolonial au sens de dépouillé de colonie, sans impérialisme, sans domination est possible sans passer par l'étape de «la décolonie ». La décolonie, c'est un mouvement, un état en branle, en action. Pour décoloniser, il faut déconstruire, se défaire comme on se défait de sa religion par un acte d'apostasie. C'est ainsi qu'est venu le titre de mon atelier/performance. Il est indispensable de critiquer, déconstruire (Apostasie) afin de passer en post-colonie. Et le moment qui précède celui où la colonie n'existera plus c'est celui de la « décolonie ». 
14 La décolonie, ce sont donc les questionnements sur les pièges du langage et les trappes de l'engagement. D'où peuvent surgir les forces qui décalent nos regards et décentrent nos opinions ? Sortir de la posture autocentrée et donc rentrer dans l'intersubjectivité, permettre une construction de nos rapports sociaux polyphoniques: tels étaient les enjeux.

15 J'ai donc demandé aux étudiants d'écrire en accordant une grande importance aux mots qu'ils employaient pour décrire la situation dans laquelle ils se trouvaient - cette décolonie - et, surtout, ce dont ils voulaient se défaire : quel était l'acte d'apostasie qu'ils posaient en tant que jeunes Européens, Afropéens en Belgique, aujourd'hui ?

L'ouvrage de Joseph Conrad mais, plus encore, la polémique sur le qualificatif qui conviendrait d'employer pour le décrire y a servi de substrat pour construire à la fois l'atelier d'écriture et la performance qui s'en est suivie. Nous nous sommes essayés, pendant deux jours, au mouvement d'apostasie et de décolonie pour arriver en postcolonie.

17 Ce travail, réalisé en étroite collaboration avec Antigone Aristidou, artiste et scénographe, a donné lieu à une restitution sur scène ${ }^{10}$ au Bozar, lieu culturel bruxellois (ancien Palais des Beaux Arts). La scénographie était composée de transparents sur lesquels nous avions imprimé des images choisies avec les étudiants, qui faisaient à la fois référence à leurs écrits propres ainsi qu'à la manière dont certains passages du livre de Conrad avaient résonné en eux. Le fleuve, le portrait officiel du roi Léopold II, la statue qui le représente sur son cheval... tout l'imaginaire qu'avait charrié la lecture du roman. C'est donc ainsi que je me suis retrouvée à nouveau à travailler sur et avec le Cœur des ténèbres de Joseph Conrad.

\section{Conclusion}

18 Je ne fais pas de lecture critique de Conrad. Je pense qu'il a des manières d'écrire intéressantes, mais ce n'est pas un écrivain qui d'une certaine manière peut me captiver, et cela d'autant plus qu'il me revient assez systématiquement par la rampe.

19 Si pour lui, l'Afrique est une sorte de figure d'altérité à laquelle il se confronte, moi, je me situe ailleurs, je ne suis pas une enfant de l'Afrique coloniale. Le Congo n'est pas autre, il n'est pas un seul exotisme qui me questionne. Le Congo, le fleuve, tout cela reste quelque chose de très concret pour moi. Le Congo, c'est moi, ce n'est pas un autre à découvrir ou à mettre en valeur...

20 C'est d'ailleurs pourquoi dans mes lectures, je reste dans les écritures contemporaines avec une attention particulière pour les auteur(e)s africain(e)s. Conrad questionne l'Europe, ses contemporains, ses filles et fils. Pas moi. Finalement, il m'apparaît que ce sont surtout les Européens qui reviennent sur sa figure, qui en font un référant - non pas que je doute qu'il puisse en être un, c'est d'ailleurs une figure majeure dans la culture des empires coloniaux - mais ce référant-là n'est pas le mien.

21 Les anciens empires coloniaux auront à se débrouiller avec leur histoire et avec les cauchemars que charrie avec brio Conrad dans son récit. En tant qu'auteure africaine, je suis souvent ramenée à son écriture, à son paradigme. Cela fait peut-être question pour certains. Et c'est certainement d'un grand intérêt, en tout cas, moi, je le mesure davantage surtout à l'écoute des autres personnes intervenues avant moi ce jour. Quoi qu'il en soit, je ne me sens pas dépositaire de Conrad. Je suis très certainement 
influencée, voire même fascinée par sa capacité littéraire à décrire, car écrire l'horreur me fascine. Mais cela s'arrête-là. En tant que congolaise, féministe, lesbienne, il y a d'autres auteurs qui m'imprègnent. Je m'intéresse à l'ici et au maintenant. Et mon écriture, ma littérature est celle - en tout cas, je souhaite qu'elle le soit - de gens ordinaires qui m'entourent. Je voudrais terminer en disant que pour moi l'écriture est un acte militant. J'écris de la fiction (nouvelle et roman) et j'écris aussi du slam. Ceci étant, à bien y regarder, Conrad et son Cour des ténèbres ont très certainement influencé l'écriture de l'un de mes premiers poèmes : «Congo Eza ». Le voici :

Le Congo est un pays... Congo Eza.

Le Congo est un pays. Sa réalité dépasse la fiction. La Belgique aussi d'ailleurs, elle est au-dessus de la réalité.

Le Congo est une diaspora, éparpillée comme des abeilles en colère. Congo Eza.

Et il faut y être fort, ne pas pleurer sur les morts, craindre l'avenir mais l'affronter avec un sans-gêne plus haut que le Ruwenzori, avec classe quoi, presque désinvolte!

Après tout la misère est un business.

Le Congo est une passion plus froide que la mer du Nord.

Le Congo est à Bangui,

Le soleil est à Juba-Soudan 500 de plus depuis dimanche

Le sarcasme est enfoui dans le sable du beach de Brazzaville

L'égalité se rit des pauvres

Le Congo est une planète et ses cratères sont des charniers. Des charniers débordant de bonnes intentions. Liberté chérie, il n'y a rien qui ne soit interdit ici. Ici c'est le paradis des nantis, tous nous sommes à la fois bourreaux et victimes, incrustés dans la tempête de nos corps, nos têtes en révolte.

Nous, nous sommes le temps, les miettes du temps qu'il nous reste après avoir été pour chacun, pour d'autres que nous: les éboueurs en fluo, les techniciennes de surface.

Nous sommes la colère. La colère qui, à force d'avoir rétréci devient implacable. La tragédie se joue sous vos yeux. Et rien, non rien ne l'arrêtera car le Congo est un pays.

Prenez-le avec tout ce qu'il comprend de minable, de grand et de fort !

Oui, nos âmes sont à vendre. Non, l'enfer n'existe pas! Non la peine n'est pas infinie.

Non, le mal n'est pas ancré. Nous ne sommes pas de petites bombes, d'innombrables sous munitions venues perturber vos quotidiens pour voir couler votre sang autant que vous sucez le nôtre. Non, bien sur que non...

Le monde est à Qunu à mille sous terre.

Le Congo est un pays... si, vraiment !

Et toutes les solutions importées et locales, tous les arrangements. Tout ça ne sont que de vulgaires cataplasmes, dérisoires, posés sur les plaies des victimes et des criminels. Que nous sommes, que je suis.

Congo Eza ${ }^{11}$.

22 Je vous remercie.

\section{NOTES}

1. Cicéron, Les Catilinaires, premier discours contre Catilina. 
2. Zamenga Batukezanga (1933-2000) est un écrivain populaire et prolifique de la République démocratique du Congo.

3. Adam Hochschild; trad. de l'américain par Marie-Claude Elsen et Franck Straschitz, Les Fantômes du roi Léopold II : un holocauste oublié, Paris, Belfond, 1998.

4. Liv Bugge a conçu l'installation You Make Me Wanna Die In The Countryside. Voir http:// nodeberlin.com/archive/liv-bugge

5. Daniel Vangroenweghe, Du sang sur les lianes, Bruxelles, Didier Hatier, coll. «Grands documents ", 1986.

6. Gilles Bibeau, «Ne pas oublier M. Kurtz. L'attrait de la sauvagerie », Anthropologie et Sociétés $\mathrm{n}^{\circ}$ 3, «Psychanalyse et anthropologie: ébranlement d'une rencontre», vol. XXXIV, 2010, p. 117-136.

7. Né au Kenya en 1938, Ngugi wa Thiong'o s'est interrogé sur la pertinence de l'usage littéraire de la langue anglaise et, à la suite de son emprisonnement et de la censure, s'est mis à écrire des romans exclusivement en Kikuyu. Ngugi wa Thiong'o, Décoloniser l'esprit, [1986] ; traduit par Sylvain Prudhomme, Paris, La Fabrique, 2011.

8. Écrivain nigérian, Chinua Achebe est notamment connu pour le célèbre Things Fall Apart, roman publié en 1958.

9. Voir https://www.bozar.be/fr/activities/124110-decolonie-apostasie---joelle-sambi-nzeba

10. Décolonie. Apostasie est le titre de la performance en deux temps conçue par Joëlle Sambi dans le cadre du séminaire de l'ERG en 2017. On en trouve une captation sur Viméo: https:// vimeo.com/213806111

11. Joëlle Sambi slame et reprend les paroles de la performance Congo Eza, une création hybride où théâtre et hip-hop se mélangent. La performance a été conçue par Joëlle Sambi, Lisette Lombé et Badibanga Ndeka, mise en scène par Rosa Gasquet et produite par l'association Lezarts Urbains. Les représentations ont eu lieu au cours du printemps 2018 et reprennent dès septembre 2018. Avec l'aimable autorisation de Joëlle Sambi.

\section{RÉSUMÉS}

Artiste polymorphe, tout à la fois romancière, poétesse et performeuse, Joëlle Sambi évoque son rapport complexe au roman de Conrad.

Joëlle Sambi is a multifaceted artist (novelist, poetess, performer). She's talking about her complicated link with the Conrad's novel.

\section{AUTEUR}

\section{JOËLLE SAMBI}

Auteure, JOËLLE SAMBI NZEBA vit entre Bruxelles et Kinshasa. Ses textes connaissent un premier succès en 2003 avec Je ne sais pas rêver. En 2005, elle occupe la $2^{\mathrm{e}}$ place du prix du Jeune Écrivain francophone pour Religion ya Kitendi. Son premier roman Le monde est gueule de chèvre est paru en 2007 aux éditions Biliki. 